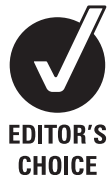

CHOICE

\title{
The temporal and nasal retinal arteriolar and venular angles in preterm infants
}

\author{
Karen Wong, ${ }^{1}$ Jeffrey $\mathrm{Ng}^{2}$ Anna Ells, ${ }^{3}$ Alistair R Fielder, ${ }^{1}$ Clare M Wilson ${ }^{4}$
}

${ }^{1}$ Department of Optometry and Visual Science, City University, London, UK

Department of Bioengineering. Imperial College London, London, UK

${ }^{3}$ Department of Surgery, Alberta Children's Hospital, Calgary,

Alberta, Canada

${ }^{4}$ Department of Visual Science, UCL Institute of Ophthalmology, London, UK

\section{Correspondence to}

Dr Clare M Wilson, Division of Visual Science, UCL Institute of Ophthalmology, 11-43 Bath Street, London EC1V 9EL, UK; clarewil25@yahoo.com

Accepted 28 August 2011 Published Online First 27 September 2011

\section{ABSTRACT}

Aim To determine the angle between the temporal or nasal retinal vessels in preterm infants and to determine the relationship of these angles to birthweight (BW), gestational age $(G A)$ and retinopathy of prematurity (ROP) status.

Methods Colour digital images were acquired during ROP screening examinations in infants born with a range of BWs and GAs between 33 and 42 weeks postmenstrual age. Four retinal vessel angles were measured: temporal venular angle, temporal arteriolar angle (tAA), nasal venular angle and nasal arteriolar angle. Measurements were performed by Computer-Aided Image Analysis of the Retina, a validated semi-automated computer software program. The relationship of each of four angles to $\mathrm{BW}$ and also to GA was determined using Mann-Whitney test and Spearman's rho, respectively. Results tAA was significantly narrower in infants with ROP and correlated positively with BW and GA. The other vessel angles, temporal venular angle, nasal arteriolar angle and nasal venular angle, showed no significant correlation with BW, GA or ROP status.

Conclusion The retinal vessel angles can be quantified in a simple repeatable manner. tAA correlated positively with $B W$ and GA, and was significantly narrower in infants with stage 3 ROP than in those without ROP or with mild disease.

\section{INTRODUCTION}

The geometrical development of vasculature adheres to Murray's principle of minimal work, ensuring that blood delivery to the tissues is achieved with optimum efficiency. ${ }^{2} 2$ The major temporal retinal vessel angle and macular position have been used to aid evaluation of the integrity of the posterior pole in the photographic grading of the Multicenter Trial of Cryotherapy for Retinopathy of Prematurity (CRYO-ROP) study ${ }^{3}$ and other randomised control clinical trials. ${ }^{4-7}$ Narrowing of the angle between the major temporal vessels has been regarded as an indicator of compromised macular function due to retinal dragging. Prediction of visual acuity in eyes with posterior pole abnormalities has been shown to be challenging. ${ }^{8}$ Despite acknowledgement of the clinical importance of the major temporal angle, to date there has only been one attempt to define its normal value ${ }^{9}$ and that study focused entirely on the temporal retinal venular angle, it did not study the arteriolar angles, nor the nasal vessels. In that study ${ }^{9}$ the mean temporal vessel (venular) angle for all gestational ages (GAs) taken together was $80.0^{\circ} \pm 17.0$ for right eyes and $80.5^{\circ} \pm 16.7$ for left eyes. A positive but not statistically significant trend was reported between the total vessel angle and increasing GA. ${ }^{9}$
In the aforementioned study by Wilson et a ${ }^{9}$ the temporal venular angle (tVA) was measured by manual methods. Recent advances in digital image analysis have permitted semi-automated measurements of the retinal vessels. These semi-automated techniques are tools to study the retinal vessel changes in infants undergoing retinopathy of prematurity (ROP) screening, but to date these studies have measured only vessel tortuosity and not retinal vessel angles. ${ }^{9-12}$ To the best of our knowledge there have been no reports quantifying the temporal arteriolar vessel and nasal vessel angles in preterm infants, or their extent of change in infants with and without ROP.

Extrapolation of Murray's hypothesis suggests that when a pathological insult challenges the blood supply to a retinal area, altering the oxygen requirements of the tissue, the vessel geometry may change to maintain the principle of minimal work. Therefore, a pathological process such as ROP could be surmised to affect the geometrical organisation of the retinal blood vessels. Other influences leading to vessel remodelling may include increased tissue perfusion and glial tissue contraction.

The objective of this study was therefore to determine whether there is any correlation between both temporal and nasal retinal vessel (arteriolar and venular) angles and various parameters (GA, birthweight (BW) and ROP stages) in preterm infants by employing a validated, quantitative, semi-automated software approach: Computer-Aided Image Analysis of the Retina (CAIAR). $^{7}$

\section{METHODS \\ Study population}

Images from preterm infants undergoing routine neonatal eye examinations at St Mary's Hospital, London, UK, and Alberta Children's Hospital, Calgary, Canada, from April 2000 to June 2004 were studied. Each baby was categorised by BW into four groups: $<750 \mathrm{~g}, \quad 751-1000 \mathrm{~g}$, $1001-1250 \mathrm{~g}$ and $>1250 \mathrm{~g}$. The GA of each infant was recorded. The stage of ROP at time of image acquisition was categorised according to the International Classification of $\mathrm{ROP}^{13}$ by the two ROP experts who obtained the images (AE, ARF). The number of clockhours of stage 3 disease and the position of the clockhours were recorded.

One digital retinal image from one screening session of preterm infants with and without ROP was acquired using a digital camera (RetCam 130; Clarity Medical System Inc., Pleasanton, California, USA). Images were taken between 33 and 42 weeks postmenstrual age (PMA) by AE or ARF. Examination of these babies was carried out solely with the 
Figure 1 The four angles measured $1 \mathrm{~A}$ Temporal venular angle (tVA). Arrowheads show point that vessel crossed periphery of image. Arrows show mid-point of vessel taken as point at which vessel crossed circle half way between centre of optic disc and periphery of image 1B Temporal arteriolar angle (tAA) 1C Nasal venular angle (nVA) 1D Nasal arteriolar angle (nAA). nAA, nasal arteriolar angle; nVA, nasal venular angle; $t A A$, temporal arteriolar angle; tVA, temporal venular angle.
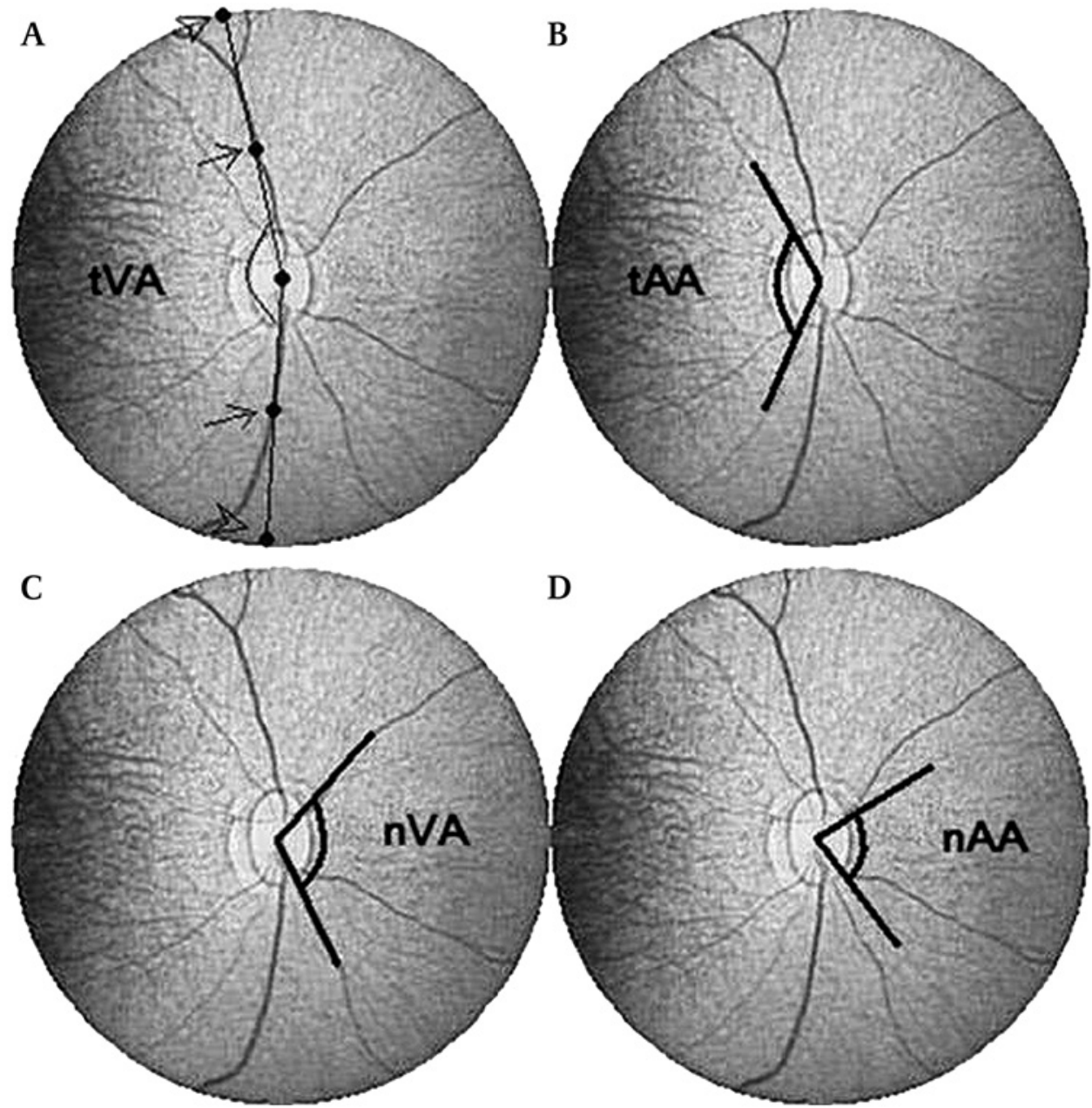

RetCam; indirect ophthalmoscopy was not used. The digital images were anonymised.

\section{Image analysis}

All images included in this study were 24 bit, red green blue (RGB) images with a size of $640 \times 480$ pixels. Each image was centred on the optic disc and cropped to 240 pixels to prevent bias from viewing peripheral ROP changes. Images were cropped using Paintshop Pro 7.0 (http://www.corel.com) and vessel angles were measured using validated novel software CAIAR. ${ }^{7}$ CAIAR uses image analysis techniques based on maximum likelihood modelfitting in a scale space framework. ${ }^{14} \mathrm{~A}$ wide-screen personal computer was used for all analyses. CAIAR generated a skeletonised image of the retinal image and identified the retinal vessels. All images were then manually edited by a single grader, who selected the vessels of interest and deselected choroidal vessels that CAIAR had mistakenly identified. The editing process took between 10 to 15 minutes per image analysed.
Each image was divided into four quadrants: superotemporal, superonasal, inferotemporal and inferonasal. The largest venule and arteriole from each quadrant were chosen manually by one observer, based on the vessel appearance, and marked. In the rare instance when a vessel was not present in a specific quadrant, the associated angle could not be calculated.

The following four angles were measured using CAIAR software: temporal arteriolar angle (tAA), tVA, nasal arteriolar angle ( $\mathrm{nAA}$ ) and nasal venular angle ( $\mathrm{nVA}$ ) (figure $1 \mathrm{~A}-\mathrm{D})$. The midpoint of each vessel (figure $1 \mathrm{~A}$, arrows) was taken as the point at which the vessel crossed the circle half way between the centre of the optic disc and the periphery of the image (figure 1A, arrowheads). It was found automatically by CAIAR. A straight line was drawn from the centre of the optic disc to the midpoint of the vessel. tVA was calculated as the angle between the midpoint of the superotemporal venule, the midpoint of the optic disc and the midpoint of the inferotemporal venule. tAA was calculated as the angle between the

Table 1 Angle measurements (degrees) without and with ROP (all stages)

\begin{tabular}{|c|c|c|c|c|c|c|c|c|}
\hline & \multicolumn{2}{|l|}{ tVA } & \multicolumn{2}{|l|}{ tAA } & \multicolumn{2}{|l|}{ nVA } & \multicolumn{2}{|l|}{ nAA } \\
\hline & Without ROP & With ROP & Without ROP & With ROP & Without ROP & With ROP & Without ROP & With ROP \\
\hline $\mathrm{n}$ & 34 & 41 & 29 & 39 & 25 & 38 & 22 & 35 \\
\hline Median & 134.62 & 125.49 & 108.19 & 95.02 & 104.92 & 112.38 & 89.63 & 89.86 \\
\hline IQR & $117.70-148.63$ & $110.42-142.29$ & $88.91-122.24$ & $81.17-111.12$ & $90.46-118.56$ & $91.58-130.69$ & $60.31-109.59$ & $81.17-98.79$ \\
\hline p Value & 0.12 & & $0.04^{*}$ & & 0.42 & & 0.82 & \\
\hline
\end{tabular}

${ }^{*} p<0.05$.

nAA, nasal arteriolar angle; nVA, nasal venular angle; ROP, retinopathy of prematurity; tAA, temporal arteriolar angle; tVA, temporal venular angle 
Table 2 Comparison of temporal vessel angles (degrees) between ROP stage groups

\begin{tabular}{|c|c|c|c|c|c|c|}
\hline \multirow[b]{2}{*}{ ROP stage } & \multirow[b]{2}{*}{$\mathbf{n}$} & \multicolumn{2}{|l|}{ tVA } & \multirow[b]{2}{*}{$\mathbf{n}$} & \multicolumn{2}{|l|}{ tAA } \\
\hline & & Median (IOR) & $\begin{array}{l}\text { Minimum value, } \\
\text { maximum value }\end{array}$ & & Median (IOR) & $\begin{array}{l}\text { Minimum value, } \\
\text { maximum value }\end{array}$ \\
\hline No ROP & 34 & $134.62(117.70-148.63)$ & $102.30,161.60$ & 29 & $107.41(88.91-122.24)$ & $60.31,154.88$ \\
\hline Stage 1 and 2 & 18 & $135.34(114.07-146.20)$ & $108.67,162.21$ & 16 & $100.82(85.09-121.51)$ & $49.10,153.40$ \\
\hline \multirow[t]{3}{*}{ Stage 3} & 23 & $121.08(107.35-137.66)$ & $86.61,159.82$ & 23 & $90.99(79.98-100.34)$ & $52.20,114.04$ \\
\hline & \multicolumn{3}{|c|}{ No ROP vs stage 1 and $2: p=0.89$} & \multicolumn{3}{|c|}{ No ROP vs stage 1 and $2: p=0.55$} \\
\hline & \multicolumn{3}{|c|}{ No ROP vs stage $3: p=0.02^{*}$} & \multicolumn{3}{|c|}{ No ROP vs stage $3: p=0.01^{*}$} \\
\hline
\end{tabular}

${ }^{*} \mathrm{p}<0.05$.

IQR, interquartile range; ROP, retinopathy of prematurity; tAA, temporal arteriolar angle; tVA, temporal venular angle.

midpoint of the superotemporal arteriole, the midpoint of the optic disc and the inferotemporal arteriole. The same rule applied to the nVA and nAA.

The effect of BW (measured in groups: $<750 \mathrm{~g}, 751-1000 \mathrm{~g}$, $1001-1250 \mathrm{~g}$ and $>1250 \mathrm{~g}$ ), GA and ROP status (with and without ROP; different stage of ROP) on each of the four vessel angles was then analysed. The position and extent of stage 3 disease was correlated with the angle measures.

\section{Statistical methods}

Analysis was performed using statistical software (SPSS V.16, SPSS Inc.). The Kruskal-Wallis test was used to compare vessel angles between the four BW groups. Spearmen's rank correlation test was used to analyse the correlation between vessel angles and GA of the infants. The Mann-Whitney $U$ test was used for comparison of vessel angles between infants with and without ROP and with different stages of ROP.

\section{RESULTS}

A total of 75 images from the right or left eyes of 75 infants were studied. At time of image acquisition, 34 infants had no ROP, nine had stage 1 ROP, nine had stage 2 ROP and 23 had stage 3 ROP. Forty-five infants were born at 24-27 weeks GA, 25 at 28-31 weeks GA, and five infants at $\geq 32$ weeks GA. Within the sample population, 20 infants weighed $<750 \mathrm{~g}, 25$ weighed $751-1000 \mathrm{~g}, 13$ weighed $1001-1250 \mathrm{~g}$ and seven weighed $>1250 \mathrm{~g}$.

The mean tVA of all the infants with and without ROP was $130.20^{\circ} \pm 19.27$, mean tAA was $109.44^{\circ} \pm 25.43$, mean $n V A$ was $109.44^{\circ} \pm 24.16$ and mean nAA was $89.13^{\circ} \pm 25.01$.

\section{Vessel angles in infants without and with ROP}

The results for vessel angles in infants without and with ROP are given in full in table 1. tAA was more acute in infants with ROP $\left(95.02^{\circ}\right)$ than those without ROP (108.19 , $\left.p<0.05\right)$. Median tVA was $125.49^{\circ}$ in infants with ROP and $134.62^{\circ}$ $(p=0.12)$ in those without ROP $(p=0.12)$. nVA and nAA were not more acute in those infants with ROP compared with those without ROP.

\section{Vessel angles with stages of ROP}

Tables 2 and 3 presents the vessel angles by ROP stage. tVA and tAA were significantly narrower in eyes with stage 3 ROP compared with those with no ROP (tVA, $\mathrm{p}=0.02$; tAA, $\mathrm{p}=0.01$ ). No significant difference was found in any of the four vessel angles between no ROP and ROP stages 1 and 2 or between stages 1 and 2 and stage 3 (tables 2 and 3 ).

\section{Clockhours of ridges involved}

Of the 75 images studied, 29 had the number and position of clockhours of disease recorded. Of these 29 images eight had $360^{\circ}$ ridge involvement, six had temporal and nasal ridges, nine had temporal ridges and six had nasal ridges.

\section{Vessel angles according to GA}

Increase in GA of the infant at birth correlated with increase in tAA $(p=0.02)$ (table 4). Increase in GA of the infant at birth did not correlate with increase in tVA or nasal vessel angles.

\section{Vessel angle according to BW}

tAA increased with increasing BW $(p<0.005)$. tAA increased with BW up to $1250 \mathrm{~g}$, then in groups with BW $>1250 \mathrm{~g}$ the angle was not greater (figure $2 \mathrm{~B}$ ). No significant increase was found between the BW groups and tVA, nVA and $\mathrm{nAA}(\mathrm{p}=0.32$, 0.56 and 0.81 , respectively) (figure $2 \mathrm{~A}-\mathrm{D}$ ).

\section{DISCUSSION}

As mentioned in the introduction, the only previous study to measure the angle of the major retinal vessels employed a manual method in which angle measurements were taken at the point along the vessel that vertically bisected the fovea. ${ }^{9}$ In the present study the angles were measured semi-automatically by CAIAR at 60 pixels from the optic disc. This enabled automatic and repeatable measures of all four vessel angles. In this study we report a tVA of $130.2 \pm 19.27$, whereas in 2006 the Wilson et al reported the tVA of right eyes to be $80 \pm 17.0$ and left eyes to be $80.5 \pm 16.7$. The angle was measured closer to the optic disc in this study ( 60 pixels from the disc centre) than in the manual study (at the level of the fovea). The familiar decrease in curvature of the retinal vessels as they become more

Table 3 Comparison of nasal vessel angles (degrees) between ROP stage groups

\begin{tabular}{|c|c|c|c|c|c|c|}
\hline \multirow[b]{2}{*}{ ROP stage } & \multirow[b]{2}{*}{$\mathbf{n}$} & \multicolumn{2}{|l|}{ nVA } & \multirow[b]{2}{*}{$\mathbf{n}$} & \multicolumn{2}{|l|}{ nAA } \\
\hline & & Median (IOR) & $\begin{array}{l}\text { Minimum value, } \\
\text { maximum value }\end{array}$ & & Median (IOR) & $\begin{array}{l}\text { Minimum value, } \\
\text { maximum value }\end{array}$ \\
\hline No ROP & 25 & $104.92(90.46-118.56)$ & $67.02,154.53$ & 22 & $89.63(60.31-109.59)$ & $40.46,143.28$ \\
\hline Stage 1 and 2 & 16 & $103.29(89.54-128.33)$ & $70.96,150.48$ & 14 & $88.00(80.43-105.52)$ & $57.96,127.42$ \\
\hline \multirow[t]{3}{*}{ Stage 3} & 22 & $114.12(99.20-130.32)$ & $61.78,161.58$ & 21 & $89.96(81.54-98.29)$ & $62.21,131.56$ \\
\hline & \multicolumn{3}{|c|}{ No ROP vs 1 and $2: p=0.83$} & \multicolumn{3}{|c|}{ No ROP vs 1 and $2: p=0.80$} \\
\hline & \multicolumn{3}{|c|}{ No ROP vs $3: p=0.30$} & \multicolumn{3}{|c|}{ No ROP vs $3: p=0.88$} \\
\hline
\end{tabular}

IQR, interquartile range; ROP, retinopathy of prematurity; $\mathrm{nAA}$, nasal arteriolar angle; nVA, nasal venular angle. 
Table 4 Correlation between vessel angles (degrees) and gestational age of infant at birth

\begin{tabular}{lllll}
\hline Vessel angle & tVA & tAA & nVA & nAA \\
\hline $\mathrm{n}$ & 75 & 68 & 63 & 57 \\
Median (IQR) & $131.98(112.21-$ & $98.53(84.13-$ & $111.92(90.46-$ & $89.86(73.17-$ \\
& $147.32)$ & $113.91)$ & $124.99)$ & $108.88)$ \\
Spearman rho & 0.15 & 0.29 & -0.12 & 0.10 \\
$\mathrm{p}$ Value & 0.21 & $0.02^{*}$ & 0.34 & 0.46 \\
\hline${ }^{*} \mathrm{p}<0.05$. & & \\
IQR, interquartile range; nAA, nasal arteriolar angle; nVA, nasal venular angle; tAA, temporal \\
arteriolar angle; tVA, temporal venular angle.
\end{tabular}

peripheral explains the smaller tVAs measured in the former manual study. 9

There are several potential limitations of this study. First, analyses were conducted on only 75 images; this may have contributed to our finding of no difference in the angles of infants with no ROP and stages 1 and 2, although it is also possible this finding is correct and will remain even with larger numbers. Second, due to small numbers in this pilot study it was not possible to fully differentiate the effect of ROP from other parameters of prematurity. Third, the inherent challenge of imaging the neonatal retina may lead to compromised picture quality and contrast, which could affect the accuracy of these measurements. We postulate, however, that vessel angle measurements are less affected by image quality and pulse cycle than measurement of vessel width. ${ }^{7} 11{ }^{15}$ Longitudinal analysis of vessel angles would be interesting to determine whether there are detectable changes in the vessel angles with GA and also progression and regression of ROP.

This study compared all four retinal vessel angles - nasal and temporal arteriolar and venular-in preterm infants and attempted to examine their association with the presence and absence of ROP, ROP stages, GA and BW. No significant correlation was found between tVA and GA (table 4), which is comparable to a previous study. ${ }^{9}$ We observed that tAA increases with increasing BW and GA. We observed that the trend of increasing tAA tailed off in groups with BW >1250 g, which may be influenced by the relatively small sample in this group. As BW and GA are the two main known risk factors for $\mathrm{ROP}$, it is not surprising that tAA is less acute in infants without ROP. From our findings, we hypothesise that the arteriolar angle is more affected by premature birth than the venular angle, and that the temporal vessel angles may be more sensitive to change than the nasal angles. Unfortunately our data regarding the position and extent of ridge involvement in clockhours were not complete. However, there appears to be a similar amount of nasal and temporal ridges, suggesting that the narrower angles seen in the temporal vessels in infants with ROP compared with those without ROP is not due to the increased disease presence in that area. Further studies looking more closely at ridge position in relation to vessel angle are warranted.

Retinal venular and arteriolar circulations are influenced by different factors. The branching structure of vessels in ROP may be influenced by the change in their diameters. Such angles may change subsequently in an attempt to provide adequate oxygenation to the pathologically affected retina that has been externally exposed to a different biochemical environment from that in utero. In terms of arteriolar circulation, apart from tAA, arterioles are also found to be narrower in children with lower weight $^{16}$ and are linked with higher cardiovascular risk in adulthood. ${ }^{17}$

Murray's law states that vessel development follows the rule of minimal energy. ${ }^{1} 218$ There is evidence that the normal topological dimension is disrupted in diseased retinae. ${ }^{15} 16$ 19-21 A recent study shows that Murray's Law may be less applicable in some conditions. ${ }^{22}$ According to Murray's law, the total volumetric flow of the vascular system is contained by a set of vasculature whose cube of the radius of a parent vessel equals the sum of the cubes of the radii of the daughter vessels. Other factors that may be responsible for retinal vessel restructuring in
Figure 2 Change of vessel angles with birthweights. BW, birthweight; nAA, nasal arteriolar angle; nVA, nasal venular angle; tAA, temporal arteriolar angle; tVA, temporal venular angle.
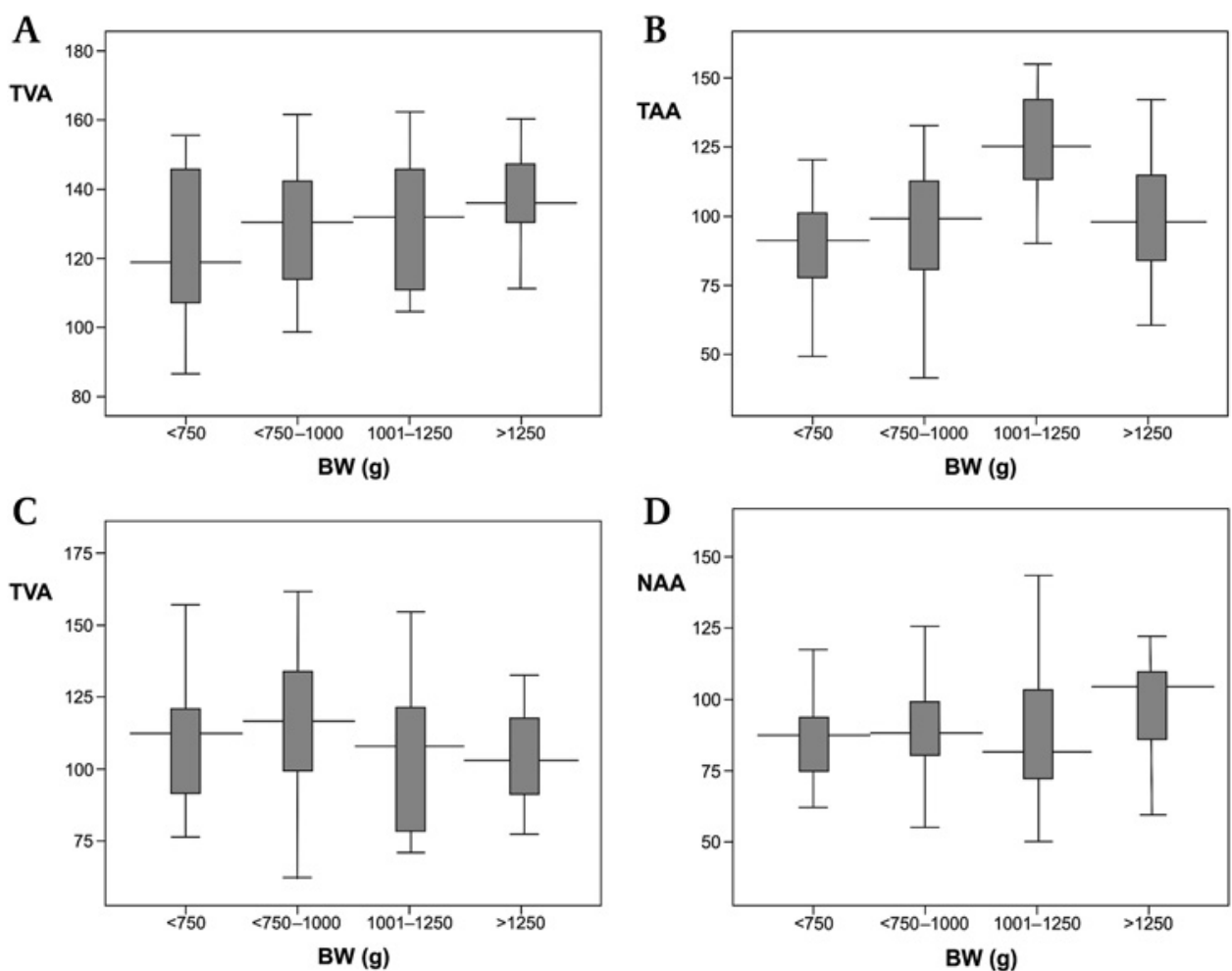
disease include remodelling secondary to increased blood flow and contraction of glial tissue along the ridge edge exerting consequent forces on the more distant retinal architecture.

Early life factors may affect vascular structure. ${ }^{23}$ There are currently several hypotheses regarding factors that may cause the changes in retinal vessel architecture, such as flow dynamics and microvascular density. ${ }^{24} 25$ Theoretically, narrower angles result in a lower vascular density, leading to a less dense, more energy-consuming retinal vascular network, as is evident in men with lower BW. ${ }^{17}$ Although we cannot extrapolate from these results directly, it is reasonable to believe that retinal vessel angle development is likely to follow this theory. However, more and larger scale studies in preterm infants will be needed to establish a clearer association between the branching patterns and ROP and its associated risk factors.

In conclusion, in this small study, we have demonstrated that the vessel angles can be measured and that tAA is significantly changed with BW, GA and ROP. A better understanding of the nature of retinal vascular architecture development may enable us to establish more accurate criteria for the diagnosis of ROP and increase the feasibility of employing tAA to aid prediction of ROP status.

\section{Competing interests None.}

Ethics approval Ethics approval was provided by St Mary's Hospital Research and Development Department and University of Calgary Conjoint Health Research Ethics Board. This study followed the tenets of the declaration of Helsinki.

Contributors ARF and CMW designed the study; ARF, AE and CMW collected clinical images; ARF, JN and CMW developed the image analysis software (in collaboration with bioengineers at Imperial College London); KW performed semi-automated image analysis; KW, ARF and CMW analysed the data; KW, ARF, AE, JN and CMW drafted and revised the paper.

Provenance and peer review Not commissioned; externally peer reviewed.

\section{REFERENCES}

1. Murray CD. The physiological principle of minimal work. The vascular system and the cost of blood volume. Proc Natl Acad Sci U S A 1926;12:207-14.

2. Murray CD. The physiological principle of minimal work II. Oxygen exchange in capillaries. Proc Natl Acad Sci U S A 1926;12:299-304.

3. Watzke RC, Robertson JE Jr, Palmer EA, et al. Photographic grading in the retinopathy of prematurity cryotherapy trial. Arch Ophthalmol 1990; 108:950-5.
4. Anon. Supplemental therapeutic oxygen for prethreshold retinopathy of prematurity (STOP-ROP), a randomized, controlled trial. I: primary outcomes. Pediatrics 2000;105:295-310.

5. Reynolds JD, Hardy RJ, Kennedy KA, et al. Lack of efficacy of light reduction in preventing retinopathy of prematurity. Light reduction in retinopathy of prematurity (LIGHT ROP) cooperative group. N Engl J Med 1998;338:1572-6.

6. Early Treatment for Retinopathy of Prematurity Cooperative Group. Revised indications for the treatment of retinopathy of prematurity: results of the early treatment for retinopathy of prematurity randomized trial. Arch Ophthalmol 2003;121:1684-96

7. Wilson CM, Cocker KD, Moseley MJ, et al. Computerized analysis of retinal vessel width and tortuosity in premature infants. Invest Ophthalmol Vis Sci 2008;49:3577-85.

8. Reynolds J, Dobson V, Quinn GE, et al. Prediction of visual function in eyes with mild to moderate posterior pole residua of retinopathy of prematurity. Cryotherapy for Retinopathy of Prematurity Cooperative Group. Arch Ophthalmol 1993;111:1050-6.

9. Wilson C, Theodorou M, Cocker KD, et al. The temporal retinal vessel angle and infants born preterm. Br J Ophthalmol 2006;90:702-4.

10. Gelman R, Jiang L, Du YE, et al. Plus disease in retinopathy of prematurity: pilot study of computer based and expert diagnosis. J AAPOS 2007:11:532-40.

11. Wallace DK, Freedman SF, Zhao Z. A pilot study using 'ROPtool' to quantify plus disease in retinopathy of prematurity. J AAPOS 2007;11:381-7.

12. Swanson C, Cocker KD, Parker KH, et al. Semiautomated computer analysis of vesse growth in preterm infants without and with ROP. Br J Ophthalmol 2003;87:1474-7.

13. The Committee for the Classification of Retinopathy of Prematurity. The international classification of retinopathy of prematurity revisited. Arch Ophthalmol 2005; 123:991-9.

14. $\mathbf{N g ~ J , ~ C l a y ~ S T , ~ B a r m a n ~ S A , ~ e t ~ a l . ~ M a x i m u m ~ l i k e l i h o o d ~ e s t i m a t i o n ~ o f ~ v e s s e l ~}$ parameters from scale space analysis. Image Vis Comput 2010;28:55-63.

15. Patton N, Maini R, MacGillivary T, et al. Effect of axial length on retinal vascular network geometry. Am J Ophthalmol 2005:140:648-53.

16. Mitchell P, Liew G, Rochtchina E, et al. Evidence of arteriolar narrowing in low birth weight children. Circulation 2008;118:518-24.

17. Chapman N, Mohamudally N, Cerutti A, et al. Retinal vascular network architecture in low birth weight men. J Hypertens 1997;15:1449-53.

18. Sherman TF. On connecting large vessels to small. The meaning of Murray's Law. J Gen Physiol 1981;78:431-53.

19. Masters BR. Fractal analysis of the vascular tree in the human retina. Annu Rev Biomed Eng 2004;6:427-52.

20. Hughes AD, Wong TY, Witt N, et al. Determinants of retinal microvascular architecture in normal subjects. Microcirculation 2009;16:159-66.

21. Liew G, Wang JJ, Cheung N, et al. The retinal vasculature as a fractal: methodology, reliability, and relationship to blood pressure. Ophthalmology 2008;115:1951-6.

22. Francis C, Frederic L, Sulvie L, et al. Scaling laws for branching vessels of human cerebral cortex. Microcirculation 2009:1:1-15.

23. Tapp RJ, Williams C, Witt N, et al. Impact of size at birth on the microvasculature: the Avon Longitudinal Study of Parents and Children. Paediatrics 2007:120:e1225-8.

24. Le Noble F, Fleury V, Pries A, et al. Control of arterial branching morphogenesis in embryogenesis: go with the flow. Cardiovasc Res 2005;65:619-28.

25. Djonov VG, Kurz H, Burri PH. Optimality in the developing vascular system: branching remodelling by means of intussusceptions as an efficient adaptation mechanism. Dev Dyn 2002;224:391-402. 